\title{
Generation of reduced macrolide analogs by regio-specific biotransformation
}

\author{
Je Won Park ${ }^{1}$, Sung Ryeol Park ${ }^{1}$, Ah Reum Han ${ }^{2}$, Yeon-Hee Ban ${ }^{1}$, Young Ji Yoo ${ }^{1}$, Eun Ji Kim ${ }^{1}$, Eunji Kim ${ }^{1}$ \\ and Yeo Joon Yoon ${ }^{1}$
}

The Journal of Antibiotics (2011) 64, 155-157; doi:10.1038/ja.2010.143; published online 24 November 2010

Keywords: 2,3-dihydro-oligomycin A; 10,11-dihydro-tilmicosin; bioconversion; Streptomyces venezuelae

The emergence of antibiotic-resistant pathogens has rekindled interest toward the discovery of new antibiotics. Semi-synthetic or biosynthetic approach using microbe as a biocatalyst can be an efficient tool for the targeted modification of existing antibiotic chemical scaffolds to create the next generation of antibiotics as well as previously uncharacterized therapeutic agents. ${ }^{1}$ The macrolide antibiotics are a group of polyketides whose activity is derived from the presence of a large macrocyclic lactone ring. They are a structurally diverse class of natural products that show a wide variety of bioactive properties, including anticancer, antifungal, immuno-suppressive and anti-aging activity. ${ }^{2}$ Oligomycin A, which was first isolated from a culture of soil bacterium Streptomyces diastatochromogenes in 1954, is a major component of oligomycins as a series of 26-membered macrocyclic lactones (Figure 1). ${ }^{3}$ This antibiotic has also been reported to possess some potential antitumor activity. ${ }^{4}$ Tilmicosin, a semi-synthetic tylosin that occurs as a 16-membered macrolide in the fermentation of $S$. fradiae, has use as a veterinary antibiotic (Figure 1$){ }^{5}$ We recently reported the unique and regio-specific hydrogenation activity toward unsaturated 12- (methymycin) and 14-membered (pikromycin) macrolides by $S$. venezuelae, and its application to the generation of unnatural 16-membered macrolides. ${ }^{6}$ This bacterial reduction system could recognize the specific structural elements around the target double bond, that is, a carbonyl functional group on the neighboring carbon and a methyl group onto another adjoining carbon in the polyketide backbone (depicted as faded rectangles in Figure 1).

In this study, an attempt was made to expand the applicability of the unique bio-hydrogenation activity of $S$. venezuelae toward other unsaturated macrolide antibiotics (natural oligomycin A and semisynthetic tilmicosin) that possess the above mentioned identical catalytic scaffold, and the structure and antibiotic potential of two previously uncharacterized macrolides, 2,3-dihydro-oligomycin A (1) and 10,11-dihydro-tilmicosin (2), isolated from a S. venezuelae culture supplemented with oligomycin A and tilmicosin, respectively, were evaluated.

A recombinant strain of $S$. venezuelae $\mathrm{YJ} 028,{ }^{6}$ which had both the pikromycin polyketide synthase-encoding gene and desosamine biosynthetic genes deleted, was provided with oligomycin A or tilmicosin. The organism was cultivated in SCM media (1.5\% soluble starch, $2.0 \%$ soytone, $0.01 \% \mathrm{CaCl}_{2}, 0.15 \%$ yeast extract, and $1.0 \% \mathrm{MOPS}$ ) in baffled Erlenmeyer flasks. After 2 days growth at $30^{\circ} \mathrm{C}$, the cultures were supplemented with either macrolides oligomycin A (Sigma, St Louis, MO, USA) and tilmicosin (Sigma) at a final concentration of $5 \mu \mathrm{g} \mathrm{ml}^{-1}$, and then incubated for additional 3 days. The whole cultures were extracted and partitioned using EtOAc, and then the organic extracts were concentrated under vacuum. The dried residues were dissolved in $200 \mu \mathrm{l}$ of $\mathrm{MeOH}$ and a portion of the solvent was subjected to HPLC-ESI-MS (Waters, Milford, MA, USA) analysis. ${ }^{6}$ Consequently, supplementation of each non-native macrolide led to $\sim 67$ and $30 \%$ conversion yields for the corresponding reduced macrolides 1 and 2, respectively (see Supplementary Figure S1). To isolate and structurally characterize both converted macrolides 1 and 2, crude extracts containing 1 were prepared from $150 \mathrm{ml}$ (three batch cultivations) of the fermentation broth from YJ028 strain fed with oligomycin A (total $0.75 \mathrm{mg}$ ), whereas crude extracts containing 2 were from $250 \mathrm{ml}$ (five batches) culture broth from the same strain supplemented with tilmicosin $(1.25 \mathrm{mg})$. These new macolides in the crude extracts were purified by chromatographic isolation using a preparative reversed-phase HPLC on a Watchers 120 ODS-BP $(250 \times 10.0 \mathrm{~mm}, 5.0 \mu \mathrm{m} \text {, Daiso, Osaka, Japan })^{6}$ and subsequent ESIMS analyses of each fraction ( $5 \mathrm{ml}$ per fraction) collected. Compound 1 was obtained as a white powder $(0.41 \mathrm{mg})$, which analyzed for the molecular formula $\mathrm{C}_{45} \mathrm{H}_{76} \mathrm{O}_{11}$ by the HR-ESI-MS $\left([\mathrm{M}+\mathrm{H}]^{+} \mathrm{m} / z\right.$ 793.5409, calcd. 793.5421) using LCT-premier XE mass spectrometer (Waters; see Supplementary Figure S2). The MS/MS spectrum of 1 at

${ }^{1}$ Department of Chemistry and Nano Science, Ewha Womans University, Seoul, Republic of Korea and ${ }^{2}$ Interdisciplinary Program of Biochemical Engineering and Biotechnology, Seoul National University, Seoul, Republic of Korea

Correspondence: Professor YJ Yoon, Department of Chemistry and Nano Science, Ewha Womans University, Seoul 120-750, Republic of Korea.

E-mail: joonyoon@ewha.ac.kr

Dedicated to the late $\mathrm{Dr} \mathrm{C}$ Richard Hutchinson for his exceptional contributions to natural product biosynthesis, engineering and drug discovery.

Received 8 September 2010; revised 25 October 2010; accepted 26 October 2010; published online 24 November 2010 


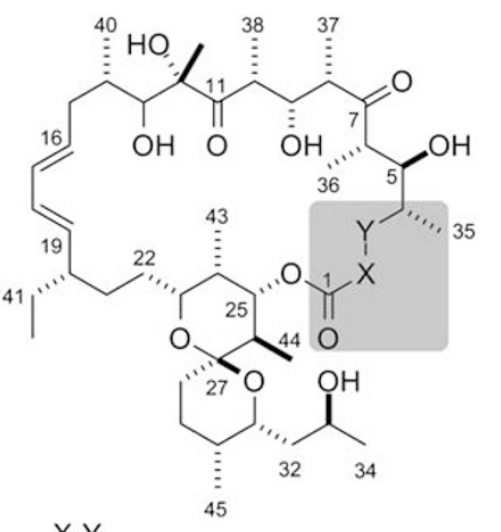

$\mathrm{X}-\mathrm{Y}$

$\mathrm{CH}=\mathrm{CH}$ oligomycin $\mathrm{A}$

$\mathrm{CH}_{2}-\mathrm{CH}_{2}$ 2,3-dihydro-oligomycin A (1)

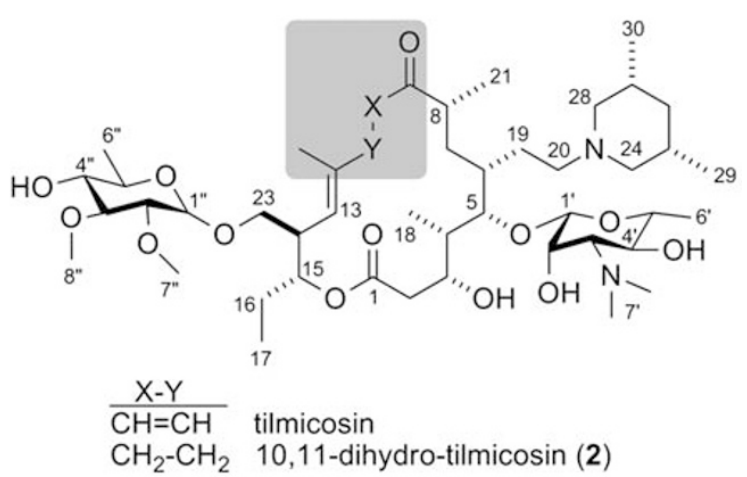

$\begin{array}{ll}\mathrm{CH}=\mathrm{CH} & \text { tilmicosin } \\ \mathrm{CH}_{2}-\mathrm{CH}_{2} & \text { 10,11-dihydro-tilmicosin (2) }\end{array}$

Figure 1 Structure of the macrolides. The faded boxes show the regio-specific functions.

$m / z 793$, which represents its protonated molecular ion, showed the main fragment ion at $\mathrm{m} / z$ 447, that is identical to that of oligomycin $\mathrm{A},{ }^{7}$ suggesting that the structural modification is likely to occur in the polyketide chain spanning from C1 to C12 position (see Supplementary Figure S3). On the other hand, HR-ESI-MS of 2 (light yellow powder; $0.30 \mathrm{mg}$ ) gave an $\mathrm{m} / \mathrm{z}$ of 871.5835 as $[\mathrm{M}+\mathrm{H}]^{+}$, which corresponds to the calculated molecular formula for $\mathrm{C}_{46} \mathrm{H}_{82} \mathrm{~N}_{2} \mathrm{O}_{13}$ (871.5850; see Supplementary Figure S2). The MS/MS spectrum of tilmicosin at $m / z 869$ indicated two fragment ions at $m / z 174$ and 696, which arise from the cleavage of a mycaminose moiety attached at C5 position, whereas MS/MS spectrum of $\mathbf{2}$ showed a similar fragment at $\mathrm{m} / \mathrm{z} 174$ as in case of tilmicosin, but the characteristic product ion at $\mathrm{m} / \mathrm{z} 698$ that is different by $2 \mathrm{Da}$ from a fragment ion at $\mathrm{m} / \mathrm{z} 696$ typical to tilmocosin, ${ }^{8}$ implying the disappearance of a double bond in the polyketide scaffold (see Supplementary Figure S3). Their chemical structures were further elucidated by ${ }^{1} \mathrm{H}$ - and ${ }^{13} \mathrm{C}-\mathrm{NMR}$ spectroscopic analyses. The NMR samples were prepared by dissolving each macrolide in $200 \mu \mathrm{l}$ of $\mathrm{CDCl}_{3}$ in a $5 \mathrm{~mm}$ Shigemi advanced NMR microtube (Sigma) matched to the solvent. Their ${ }^{1} \mathrm{H}$ - and ${ }^{13} \mathrm{C}-\mathrm{NMR}$ spectra were acquired using a Varian INOVA 500 NMR spectrometer (Varian, Inc., Palo Alto, CA, USA) at $298 \mathrm{~K}$. The chemical shifts of each converted macrolide were assigned by a comparison with those of the parent macrolides oligomycin A and tilmicosin. Compounds 1 and 2 were identified as 2,3-dihydro-oligomycin A and 10,11-dihydro-tilmicosin, respectively (see Supplementary Tables S1 and S2).

The most obvious change observed by a comparison of the ${ }^{1} \mathrm{H}$ NMR spectroscopic data of oligomycin A and its reduced product 1 was the absence of signals at 5.83 and 6.88 p.p.m., which are typical for olefinic protons (H-2,3) in oligomycin A. The upfield shift of the C-2 and C-3 signals of the parent compound (from 121.5 and 147.6 p.p.m. to 30.6 and 25.3 p.p.m., respectively) also support the distinction found in the above ${ }^{1} \mathrm{H}-\mathrm{NMR}$ data, demonstrating that $\mathbf{1}$ is a reduced form of oligomycin A with an absent C-2,3 double bond (see Supplementary Table S1, Figures S4 and S6). Similarly, a noticeable difference between tilmicosin and its reduced form 2 was observed at the C-10,11 position. Indeed, the findings of both the absence of a signal downfield of 6.00 p.p.m. in the ${ }^{1} \mathrm{H}-\mathrm{NMR}$ spectroscopic data of 2 , and the upfield shift of signals designating C-10 and C-11 positions in tilmicosin, indicated 2 to also be a reduced form of semi-synthetic tilmicosin (see Supplementary Table S2, Figures S5 and S7). These results show that the reduced macrolides $\mathbf{1}$ and $\mathbf{2}$ are transformed by
Table 1 Comparison of the MIC data for compounds 1,2 , and the corresponding oligomycin A and tilmicosin

\begin{tabular}{ccccc} 
& Oligomycin A & $\mathbf{1}$ & Tilmicosin & $\mathbf{2}$ \\
\hline MIC $\left(\mu \mathrm{g} \mathrm{ml}^{-1}\right)^{\mathrm{a}}$ & 25.0 & 12.5 & 1.56 & 1.56 \\
\hline
\end{tabular}

Abbreviations: 1, 2,3-dihydro-oligomycin A; 2, 10,11-dihydro-tilmicosin.

aoligomycin A and $\mathbf{1}$ were tested against Saccharomyces cerevisiae, whereas tilmicosin and $\mathbf{2}$ against Bacillus subtilis.

S. venezuelae, which contains regio-selective activity for the biohydrogenation ${ }^{6}$ of diverse ring macrolides. A recent study reported the chemical modification of oligomycin A, yielding pyrazolo (1, 5-a) pyridine-conjugated oligomycin A. ${ }^{9}$ However, there are no reports on the generation of oligomycin analogs by biotransformation.

As each macrolide oligomycin A and tilmicosin was initially discovered as an antifungal and antibacterial (especially against Gram-positive bacteria) antibiotic, respectively, ${ }^{3,5}$ the antimicrobial activities of compounds $\mathbf{1}$ and $\mathbf{2}$ against Saccharomyces cerevisiae and Bacillus subtilis, respectively, were determined using the microdilution method recommended by the Clinical and Laboratory Standard Institute (formerly National Committee for Clinical Laboratory Standards). ${ }^{10}$ Briefly, S. cerevisiae ATCC 9763 and B. subtilis ATCC 23857 were grown in Antibiotic 19 (Difco, BD Biosciences, San Jose, CA, USA) and Mueller-Hilton broth (Difco), respectively. Oligomycin A and its corresponding 1 were tested against $S$. cerevisiae, whereas tilmicosin and 2 was tested against $B$. subtilis. The growth of test strains was monitored at $600 \mathrm{~nm}$ using a Labsystems Bioscreen $C$ reader (Labsystems Corp., Helsinki, Finland), and the MIC was determined to be the lowest concentration of the macrolides diluted in a broth medium that inhibited the growth of the test microorganisms.

Reduced macrolide $\mathbf{1}$ showed a twofold increase in activity against S. cerevisiae ( $\mathrm{MIC} \sim 12.5 \mu \mathrm{g} \mathrm{ml}^{-1}$ ) compared with oligomycin A (MIC $\sim 25.0$ ), and compound 2 was as active as tilmicosin (Table 1). Therefore, the lack of these double bonds in both macrolides has no adverse effects on their antibiotic activities. Evidently, this is the first report on the microbe-derived biotransformation of oligomycin A and tilmicosin, even though the antibacterial activity of the reduced macrolide 2 was not improved. In our previous works, we determined that the hydrogenation activity exerted by $S$. venezuelae is not common among the representative streptomycetes, and also found 
that this unique bio-hydrogenation function is independent of reductive enzymatic domains (that is, enoyl reductase) in the modular-type polyketide synthase system including the innate pikromycin polyketide synthase. ${ }^{6}$ Recent discoveries of trans-acting enoyl reductase function in several microorganisms could be a clue to identify this unusual reductive enzyme in $S$. venezuelae. ${ }^{11-13}$ In summary, diverse macrolide scaffolds, which include 26 -membered ring macrolide as well as 12-, 14-, and 16-membered ones, ${ }^{6}$ could be produced by exploiting nature's chemical diversity, demonstrating the expandability of the bio-hydrogenation activity of $S$. venezuelae to develop more diverse therapeutic resources. An attempt to expand the applicability of this unique biocatalyst toward acyclic polyketides, in which the described catalytic scaffolds are well conserved, is currently in progress.

\section{ACKNOWLEDGEMENTS}

This work was supported by the National Research Foundation of Korea grants funded by the Korea government (MEST; R0A-2008-000-20030-0, 20090073043, and 2010-0001487), and Technology Development Program (20100623) for Agriculture and Forestry, Ministry for Food, Agriculture, Forestry and Fisheries, Republic of Korea.

1 Fischbach, M. A. \& Walsh, C. T. Antibiotics for emerging pathogens. Science $\mathbf{3 2 5}$ 1089-1093 (2009).
2 Saleem, M. et al. Antimicrobial natural products: an update on future antibiotic drug candidates. Nat. Prod. Rep. 27, 238-254 (2010).

3 Smith, R. A., Peterson, W. H. \& McCoy, E. Oligomycin, a new antifungal antibiotic. Antibiot. Chemother. 4, 962-970 (1954).

$4 \mathrm{Li}, \mathrm{Y}$. C. et al. Mitochondria-targeting drug oligomycin blocked P-glycoprotein activity and triggered apoptosis in doxorubicin-resistant HepG2 cells. Chemotherapy 50, 55-62 (2004).

5 Debono, M. et al. Synthesis and antimicrobial evaluation of 20-deoxo-20-(3,5dimethylpiperidin-1-yl)desmycosin (tilmicosin, EL-870) and related cyclic amino derivatives. J. Antibiot. 42, 1253-1267 (1989).

6 Park, J. W. et al. Exploiting the natural metabolic diversity of Streptomyces venezuelae to generate unusual reduced macrolides. Chem. Commun. 44, 5782-5784 (2008).

7 Wagenaar, M. M., Williamson, R. T., Ho, D. M. \& Carter, G. T. Structure and absolute stereochemistry of 21-hydroxyoligomycin A. J. Nat. Prod. 70, 367-371 (2007).

8 Ding, J., Ren, N., Chen, L. \& Ding, L. On-line coupling of solid-phase extraction to liquid chromatography-tandem mass spectrometry for the determination of macrolide antibiotics in environmental water. Anal. Chim. Acta. 634, 215-221 (2009).

9 Lysenkova, L. N., Turchin, K. F., Danilenko, V. N., Korolev, A. M. \& Preobrazhenskaya, M. N. The first examples of chemical modification of oligomycin A. J. Antibiot. 63, 17-22 (2010).

10 NCCLS (National Committee for Clinical Laboratory Standards). Methods for Dilution Antimicrobial Susceptibility Tests for Bacteria That Grow Aerobically; Approved Standard M7-A5 (NCCLS, Wayne, PA, 2000).

11 Siméone, R., Constant, P., Guilhot, C., Daffé, M. \& Chalut, C. Identification of the missing trans-acting enoyl reductase required for phthiocerol dimycocerosate and phenolglycolipid biosynthesis in Mycobacterium tuberculosis. J. Bacteriol. 189, 4597-4602 (2007).

12 Halo, L. M. et al. Authentic heterologous expression of the tenellin iterative polyketide synthase nonribosomal peptide synthetase requires coexpression with an enoyl reductase. Chembiochem. 9, 585-594 (2008).

13 Bumpus, S. B., Magarvey, N. A., Kelleher, N. L., Walsh, C. T. \& Calderone, C. T. Polyunsaturated fatty-acid-like trans-enoyl reductases utilized in polyketide biosynthesis. J. Am. Chem. Soc. 130, 11614-11616 (2008).

Supplementary Information accompanies the paper on The Journal of Antibiotics website (http://www.nature.com/ja) 